\title{
A Low-cost Neural-based Approach for Wood Types Classification
}

\author{
Ruggero Donida Labati, Marco Gamassi, Vincenzo Piuri IEEE Fellow, Fabio Scotti IEEE Member \\ University of Milan, Department of Information Technologies \\ Bramante 65, 26013 Crema (CR), Italy \\ \{rdonida, mgamassi, fscotti, piuri\}@dti.unimi.it
}

\begin{abstract}
In many applications such as the furniture and the wood panel production, the classification of wood kinds can provide relevant information concerning the aspect, the properties and the preparation procedures of the products. Usually, the wood kind classification is made by trained operators, but this solution suffers of important drawbacks: it is time consuming and it has low repeatability/accuracy since the classification is related to the operator experience and fatigue. In the literature, some attempts to solve this applicative problem by automatic systems are present, but, unfortunately, these solutions present complex measures and setups. In this paper, we present a novel approach for wood kinds classification based on a neural network system which exploits the emitted spectrum of the wood samples filtered with a bank of low-cost optical filters coupled with a set of photo detectors. The structure of the proposed system can be directly implemented in an embedded low-cost system. The results of the system simulations are very satisfactory and they demonstrate that this approach is feasible and very promising.
\end{abstract}

Index Terms-Wood kinds classification; neural classification systems; spectrum analysis.

\section{INTRODUCTION}

The classification of the wood kinds is a valuable task processed in several application related to the furniture and wood panel production [1]. The identification of the wood kind is important since it is related to different aspects, properties and costs of the materials. For instance, the minimum quantity of glue required to produce a wood panel with specific mechanical properties is related to the type of the woods used in the production, as well as the preparation procedures. Similarly, in the paper production, the wood kind is correlated to the final quantity of the paper cellulose and hence to the quality of the paper [2].

Experienced operators are usually employed to control and classify the wood kind by manual observation of the slices and the surfaces of the material. Unfortunately, this task is not fast, and the accuracy of the results is far to be standard because it depends on the fatigue and the experience of the single operator. Also chemical tests are available, but they can be processed only on samples of the production and they typically have a relevant cost in time and money.

In this work, we describe a novel approach based on neural networks capable to achieve the real-time classification of the wood kinds in industrial environments. The presented approach exploits the analysis of the wood fluorescence spectrum by means of a bank of low-cost optical filters, photo detectors and a neural network classification system. This approach offers a low-cost setup with respect to the proposals that are available in the literature. In particular, no spectrometers and complex processing systems are required: all the modules of the present approach can be easily implemented in a custom circuit or embedded in existing systems. The proposed approach has been verified by detailed simulations, starting from real spectra acquired by a broad variety of wood types. The simulations results indicate a remarkable classification capability of the proposed approach. Tests have been made in classification problems encompassing up to 21 different wood kinds.

The paper is structured as follows: in the next section the state of the art is presented and in section III the proposed approach is then detailed and discussed. In section IV, we describe the creation of the test datasets, and the design of the classifiers, then it is commented the overall classification accuracy of the presented approach.

\section{PREVIOUS WORK}

The specific spectral fingerprint of the of wood types can be analyzed by exploiting different techniques. The more common methods in the literature consider the vibrational spectroscopy (near-infrared range (NIR) [3], mid-infrared (MIR) range [4], [5], and Raman spectroscopy [6], [7]). Differently, fluorescence spectroscopy can also be considered. The vibrational spectroscopy offers a broad richness of details of the wood spectrum, but it is costly and it is difficult to be implemented in real-time systems, especially in the industrial applications. For example, important biases of the IR spectrum measures can be related to environmental noises, such as the humidity, artifacts, and presence of other thermal sources (which are very difficult to be controlled in the industrial environment).

Differently, the fluorescence spectroscopy works in a wavelength range which is more close to the visible region and the devices can offer higher SNR that the previous case, as well as a minor dependence to the environmental biases. Fluorescence spectroscopic systems have a limited cost (about 1000 euro for an entry-level device) and they can work with simple excite sources such as the diode-pumped solid state lasers.

In [8] a prototype system has been presented, where a miniature spectrometer and a frequency doubled diode-pumped solid state laser are adopted. The spectra acquired by the 
spectrometer has been processed in order to extract a proper set of features which has been classified with different inductive classification systems, such as the Linear Bayes Normal Classifier and the k-Nearest Neighbor Classifier. Results indicate that the fluorescence approach is feasible to solve the problem of the wood kind classification.

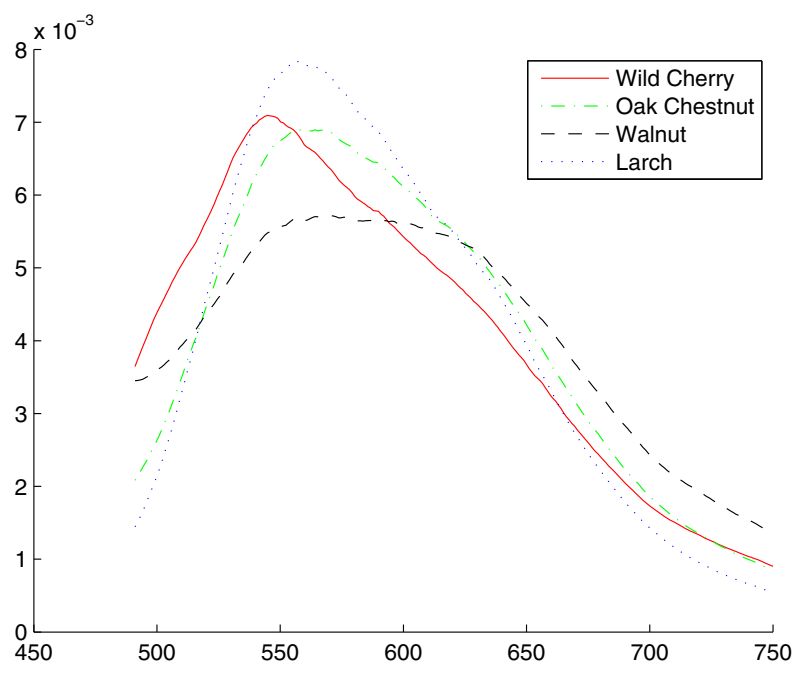

Fig. 1. Examples of inputs spectra.

\section{THE PROPOSED SYSTEM}

In this paper we present a novel approach based on the analysis of the florescence spectrum achieved without the usage of any spectrometer and by exploiting the learning capability of the neural network models.

As presented in [8], a simple exciting laser beam with an optical power limited to $50 \mathrm{~mW}$ (working in the wavelength of $473 \mathrm{~nm}$ ) can be used to produce the emitted fluorescence of the wood sample. The working hypothesis is based on the fact that the emitted spectra of different woods have different spectral patterns.

For instance, in Fig. 1 the input spectra of the wood types Wild Cherry, Oak Chestnut, Walnut and Larch are plotted in the most significant part which can qualitatively estimated in the wavelength range starting from $490 \mathrm{~nm}$ to $750 \mathrm{~nm}$. In this paper, we aim to demonstrate that the florescence spectra of the different wood kinds are distinctive patterns and they can be classified with good accuracy with neural networks systems.

\section{A. Components of the system}

Fig. 2 plots the structure of elements of the proposed approach.The diode-pumped solid state laser induces the florescence emission from the wood sample which is filtered by a set of $N$ optical filters working at different cut off wavelengths. Coupled with the filters, $N$ photodiode detectors collect the input optical power and they convert it into a $N$ different current signals that can be used as input for a neural network module.

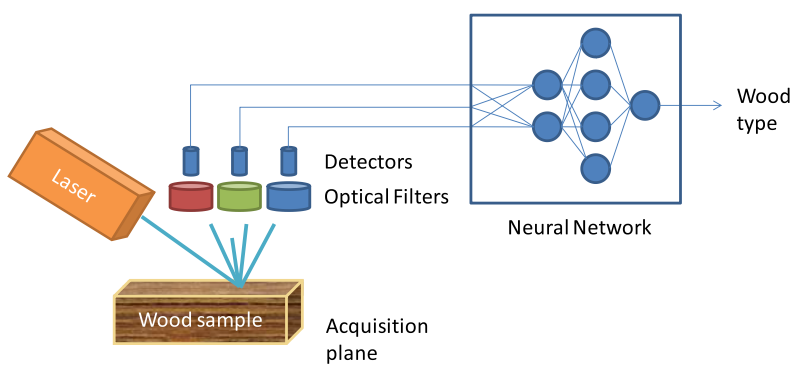

Fig. 2. Scheme of the proposed approach.

\section{B. The optical filter bank}

The market offers a great variety of optical filters with very different specifications and costs. For instance, in Fig. 3 it has been plotted the transmittance of commercial optical long wave pass filter of the following producer [9]. In this case, the transmittance curves are very vertical, with transmittance values over the transition frequency higher than 0.99 .

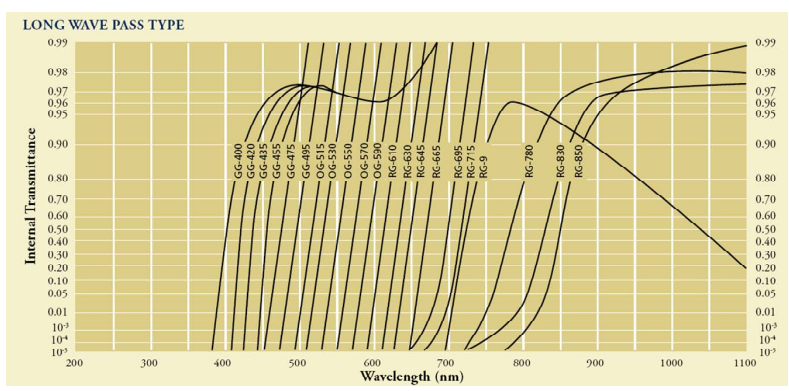

Fig. 3. Examples of curves for long wave pass type optical filters transmittance of producer CI VA REF.

The proposed system can work also with less performing optical filters, for example with transmittance curves similar to the ones shown in Fig. 4, but the classification accuracy can decrease, as described in the experimental results section. Optical filters with similar transmittance curve can be found on the market with relatively low prices (few tens of euro) [10].

Without loss of generality, in our work we simulate the filter transmittance with the following equation

$$
f(x)=\left\{\begin{aligned}
S_{b}, & \text { if } x<\lambda_{S} \\
P_{b}, & \text { if } x>\lambda_{P} \\
m_{1} x+q_{1}, & \text { if } \lambda_{S} \geq x \leq \lambda_{C} \\
m_{2} x+q_{2}, & \text { if } \lambda_{C} \geq x \leq \lambda_{P}
\end{aligned}\right.
$$

where $m_{1}=\left(S_{b}-0.5\right) /\left(\lambda_{S}-\lambda_{C}\right), q_{1}=S_{b}-m_{1} \cdot \lambda_{S}$, $m_{2}=\left(P_{b}-0.5\right) /\left(\lambda_{P}-\lambda_{C}\right)$ and $q_{2}=P_{b}-m_{2} \cdot \lambda_{P}$.

In our approach, we applied in (1) the transmittance parameters of of-the-shelf optical filters available in the market of differents cost and performances.

For example, having in input the spectrum coming from a wild cherry wood like plotted in Fig. 5a, and applying the 


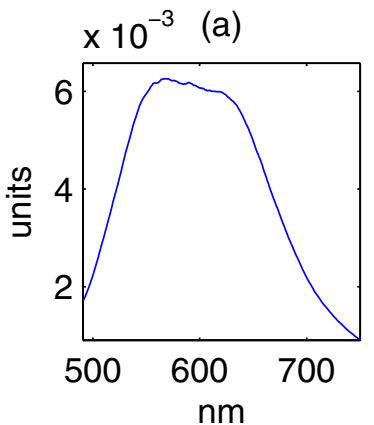

(b)

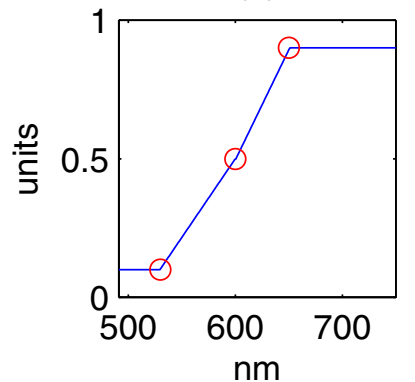

(c)

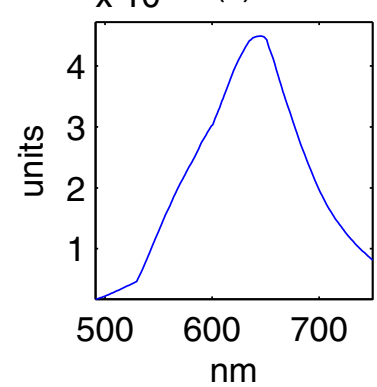

Fig. 5. Example of optical filtering. The input spectrum (a) is filtered with an optical filter with cut-off wavelength $\lambda_{C}$ equal to 600 , stop-band/pass-band wavelength limits equal to 530 and 650 respectively, and the stop-band/pass-band transmittances equal to 0.1 and 0.9 respectively. The subplot (c) reports the simulated spectrum in input to the photodetector coupled with the optical filter.

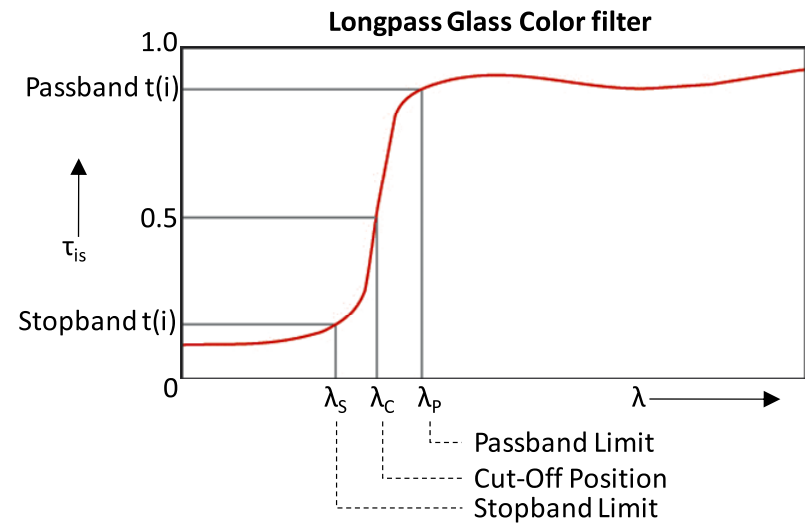

Fig. 4. Example of optical transmittance of a low-cost optical filter with its main parameters.

transmittance of a long pass filter with $\lambda_{S}=530, \lambda_{C}=600$ and $\lambda_{P}=650$ (Fig. 5b), we obtain the filtered spectrum plotted in Fig. 5c. At this stage of the work, we assume an ideal photodetector (with a flat $100 \%$ efficiency for all wavelengths). In a more refined model, of course it is possible to take into account the specific efficiency spectral curve of the chosen photodetector device.

The photodetectors hence transduce the optical energy filtered by the optical filters in the signal adapt to be processed by the neural network. More in detail, the filtering effect of the bank of filters represents an integral operation of the spectral energy in different bands of the wood fluorescence.

\section{The neural classification system}

Unfortunately, the relationship between the energy collected by the detectors and the wood types is not known, hence it is not possible to directly design an algorithm for the classification system. In this case, only the approaches based on a set of reference spectra are realistically feasible. In particular, the capability of the neural networks to correctly map input-output relationships starting from an example dataset (a set of classified wood spectra) can be here exploited to create a neural classification system. Without loss of generally, in the following we will focus on the application of the feedforward neural networks.

Notably, it is possible to consider the optical bank of filters of the proposed system (Fig. 2) as the first input layer of the neural network. This parallelism is possible since the optical filters coupled with the photodetectors process the input spectrum in a similar manner how the neural networks' neurons process their inputs (Fig. 6). Moreover, the transmittance functions of the optical filters can be very similar to the classical log-sigmoidal activation function of a neuron. In the same fashion, the optical filters coupled with the photodetectors achieve the non-linear processing of the input spectra.
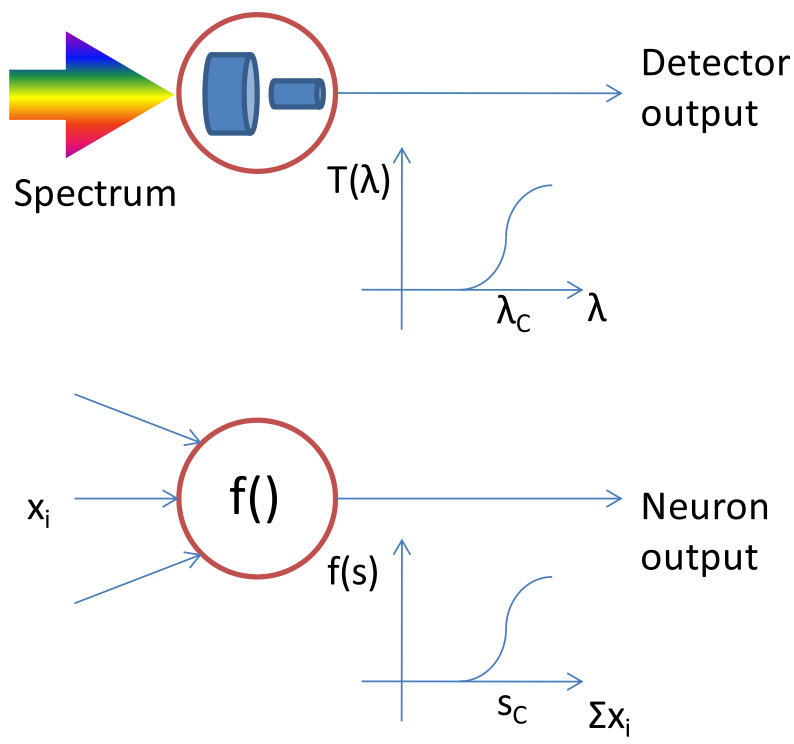

Fig. 6. Comparison of a classical neuoron attivation function with respect to an optical filter trasmittance.

Under this assumption, it is possible to consider the optical filters and the coupled photodetectors as the input layer of the subsequent neural network and consider their output as input to a hidden layer of the whole neural network. Working in this framework, the optimization of the complete neural system can regard also the parameters of the optical bank of 
filters. More simply, it is also possible to chose a normal set of filters working in the fluorescence range at different cut-off wavelengths, and then separately train the subsequent neural network. In the following we discuss the latter approach.

\section{EXPERIMENTAL RESULTS}

In this section, we apply the proposed approach by considering the real fluorescence emissions of different wood samples. At the current stage of the work, we estimated the output of the detectors by a simple simulation, taking into account the real wood spectra and the real transmittances of commercial low-cost optical filters. We assume that the outputs of the photodetectors are proportional to the energy which is present in the spectrum of the wood fluorescence filtered by the relative optical filters.

\section{A. The spectra dataset}

The spectra dataset has been created by using a set of the 21 different wood types (of certified origin purchased at Woodtechnology Gmbh) belonging the most common species. Twenty spectra for each sample have been acquired in different points by moving the samples under irradiation. During the measurements, we ensured to probe all wood zones namely heartwood, sapwood, and growth ring. The 21 wood types belonging to the dataset are the following: (1) Wild Cherry; (2) Oak Chestnut; (3) Walnut; (4) Larch; (5) Wild Pear Tree; (6) Poplar; (7) Cembar Pine ; (8) Beech Tree; (9) Alnus incana; (10) Linden Tree; (11) Alnus incana; (12) Scots Pine; (13) Oak tree; (14) Spruce; (15) Maple; (16) Taxus baccata; (17) Elm; (18) Silver Fir; (19) Birch Tree; (20) Black Locust; (21) Carpinus betulus. All spectra have been considered in the range from $490 \mathrm{~nm}$ to $750 \mathrm{~nm}$, since in this range of wavelengths the major peculiarity between the different wood fluorescence patterns are present. The adopted laser is a powertunable diode-pumped solid state $50 \mathrm{~mW}$ laser working in the wavelength of $473 \mathrm{~nm}$.

\section{B. The optical filters}

Since the market offers a plethora of different models of optical filters, we adopted a commercial set of filters which are particular chip and common from the following producer [10]. In our approach, we design the central cut-off wavelengths of the filters with equally distances in the wavelengths range $490 \mathrm{~nm}$ to $750 \mathrm{~nm}$, and we simulate the real transmittance curves by using (1). In the following, we consider four configurations of the filter banks:

- Filter Bank A - Three optical filters starting at the cut-off wavelength $\lambda_{C}$ equal to $550 \mathrm{~nm}, 600 \mathrm{~nm}$ and $650 \mathrm{~nm}$, and with stop-band/pass-band and wavelength limits equal to $\lambda_{C}-50 \mathrm{~nm}$ and $\lambda_{C}+50 \mathrm{~nm}$, and stop-band/pass-band transmittance equal to 0.1 and 0.9 respectively;

- Filter Bank B - Five optical filters with transmittance characteristics equal to Filter Bank 1, but starting at cutoff wavelengths $\lambda_{C}$ regularly spaced in the range starting from $500 \mathrm{~nm}$ to $700 \mathrm{~nm}$;
- Filter Bank C - Eleven optical filters with transmittance characteristics equal to Filter Bank 1, but starting at cutoff wavelengths $\lambda_{C}$ regularly spaced in the range starting from $500 \mathrm{~nm}$ to $750 \mathrm{~nm}$;

- Filter Bank D - Thirty optical filters with very good optical transmittance characteristics with cut-off wavelength $\lambda_{C}$ regularly spaced in the range starting from $500 \mathrm{~nm}$ to $700 \mathrm{~nm}$ and with the stop-band/pass-band wavelengths equal to $\lambda_{C}-50 \mathrm{~nm}$ and $\lambda_{C}+50 \mathrm{~nm}$ respectively, and stop-band/pass-band transmittances equal to 0.01 and 0.99 respectively.

\section{The creation and the test of the neural system}

The first classification problem we considered (Problem A) is the binary classification between the conifer and broadleaved wood spectra. This problem is related to the fact that, in some specific applications such as the wood panel production, the properties of the wood types belonging to the same class (conifer or broad-leaved) can be considered as similar. The second problem we considered (Problem B) is the classification of the 21 different wood types. Problem B can be considered as more difficult than Problem A, since the number of classes is 10 time more.

In order to prove the feasibility of the proposed approach, we considered the implementation of two-layer feed-forward neural networks with a different number of the neurons in the hidden layer. In particular, we consider log-sigmoidal activation functions for the hidden layer and simple linear function for the output neuron, using the back propagation learning method.

The classification error of the cited neural networks and its standard deviation has been estimated using the crossvalidation technique (using 12 rotations). All tests have been performed using a Pentium $1,7 \mathrm{GHz}, 1 \mathrm{~GB}$ RAM, using Windows XP Professional. The whole system has been implemented in Matlab by exploiting the available Toolboxes. The simulation results are given in Table I and II.

TABLE I

CONIFER / BROAD-LEAVED

\begin{tabular}{lcccccc}
\hline Problem A & \multicolumn{2}{c}{ FF 3 neurons } & \multicolumn{2}{c}{ FF 10 neurons } & \multicolumn{2}{c}{ FF 30 neurons } \\
& Err & Std & Err & Std & Err & Std \\
\hline Filter Bank A & 0.076 & 0.096 & 0.036 & 0.033 & 0.040 & 0.038 \\
Filter Bank B & 0.045 & 0.064 & 0.043 & 0.031 & 0.031 & 0.040 \\
Filter Bank C & 0.038 & 0.037 & 0.021 & 0.030 & 0.029 & 0.020 \\
Filter Bank D & 0.057 & 0.081 & 0.033 & 0.032 & 0.026 & 0.034 \\
\hline
\end{tabular}

The best accuracy can be found for Problem A with minimum classification error of $2.1 \%$ (Filter Bank C) with a standard deviation of 0.03 , while Problem B has a minimum error of $17.6 \%$ (Filter Bank D) with a standard deviation of 0.098 . Hence, problem $\mathrm{A}$ can be solved by using low cost optical filters $\left(\lambda_{P}-\lambda_{S} \approx 100 \mathrm{~nm}\right)$, while Problem B can be faced only by using a more expansive setup. In fact, the best results for Problem B have been obtained with thirty optical 
TABLE II

21 WOOD TYPES

\begin{tabular}{lcccccc}
\hline Problem A & \multicolumn{2}{c}{ FF 3 neurons } & \multicolumn{2}{c}{ FF 10 neurons } & \multicolumn{2}{c}{ FF 30 neurons } \\
& Err & Std & Err & Std & Err & Std \\
\hline Filter Bank A & 0.4549 & 0.055 & 0.637 & 0.058 & 0.604 & 0.050 \\
Filter Bank B & 0.4681 & 0.041 & 0.605 & 0.083 & 0.583 & 0.077 \\
Filter Bank C & 0.4681 & 0.039 & 0.586 & 0.052 & 0.597 & 0.094 \\
Filter Bank D & 0.4569 & 0.060 & 0.554 & 0.097 & 0.176 & 0.098 \\
\hline
\end{tabular}

filters characterized by good optical transmittance characteristics. Further improvements should be probably found by a fine tuning of parameters of the filter banks or adopting different optical filters.

The simulations show that this approach to wood classification is feasible and it has a limited hardware complexity. Further cost reduction can be gained by considering the usage of specific optics like the Linear Variable Bandpass Filters [9] which permit to emulate a complete set of optical filters in one single filter slab.

\section{CONCLUSiON}

The paper presented a novel approach based on neural network classification system for wood type classification. The proposed system processes the emitted fluorescence spectrum of the wood samples with a bank of low-cost optical filters and a neural network. The peculiar characteristics of the proposed approach allow for the implementation in embedded and realtime systems with a limited hardware cost. The results of the system simulations are very satisfactory and they demonstrate that that approach is feasible and promising.

\section{REFERENCES}

[1] S. Radovan, P. George, M. Panagiotis, G. Manos, A. Robert, and D. Igor, "An approach for automated inspection of wood boards," vol. 1, 2001, pp. 798-801 vol.1.

[2] H. Furumoto, "Method and device for process control in cellulose and paper manufacture," United States Patent 6398914.

[3] S. Tsuchikawa, Y. Hirashima, Y. Sasaki, and K. Ando, "Near-infrared spectroscopic study of the physical and mechanical properties of wood with meso- and micro-scale anatomical observation," Appl. Spectrosc. vol. 59, no. 1, pp. 86-93, 2005.

[4] M. H. Nuopponen, G. M. Birch, R. J. Sykes, S. J. Lee, and D. Stewart, "Estimation of wood density and chemical composition by means of diffuse reflectance mid-infrared fourier transform (drift-mir) spectroscopy," Journal of Agricultural and Food Chemistry, vol. 54, no. 1, pp. 34-40, 2006.

[5] C. R. Orton, D. Y. Parkinson, P. D. Evans, and N. L. Owen, "Fourier transform infrared studies of heterogeneity, photodegradation, and lignin/hemicellulose ratios within hardwoods and softwoods," Appl. Spectrosc., vol. 58, no. 11, pp. 1265-1271, 2004.

[6] B. K. Lavine, C. E. Davidson, A. J. Moores, and P. R. Griffiths, "Raman spectroscopy and genetic algorithms for the classification of wood types," Appl. Spectrosc., vol. 55, no. 8, pp. 960-966, 2001.

[7] H. Yang, I. R. Lewis, and P. R. Griffiths, "Raman spectrometry and neural networks for the classification of wood types. 2. kohonen self-organizing maps," Spectrochimica Acta Part A: Molecular and Biomolecular Spectroscopy, vol. 55, no. 14, pp. 2783 - 2791, 1999.

[8] P. Camorani, M. Badiali, D. Francomacaro, and M. Gamassi, "A classification method for wood types using fluorescence spectra," May 2008, pp. 1312-1315.

[9] A. Corporation, "http://www.andovercorp.com," Salem, USA.

[10] E. Optics, "http://www.edmundoptics.com," Barrington, USA. 